\title{
Antibiotic susceptibility pattern of uropathogens from some selected hospitals in Kano-Nigeria
}

\begin{abstract}
Urinary tract infection (UTI), is a common disease of public health concern that warrants frequent hospital visit by the affected patients worldwide, leading to increased health care expenditure across the globe. Knowledge of the sensitivity or otherwise of its associated pathogens to the commonly used drugs, is of paramount importance. The study was to isolate the common Urinary Tract Pathogens and to determine their pattern of susceptibility to the commonly used anti microbial agents. The study involved 300 urine samples of UTI suspects, of which 114(38\%) were positive with different types of uropathogens isolated. The isolated agents were E. coli, Klebsiella puemoniae, Staphylococcus saprophyticus, Staphylococcus aueus, Proteus mirabilis, and Pseudomonas. Various patterns of susceptibilities were observed when interacted with the commonly used antimicrobial agents, Augumentin $(30 \mu \mathrm{g})$, Nitrofurontoin $(200 \mu \mathrm{g})$, Amoxycillin $(25 \mu \mathrm{g}), \quad$ Tetracyclin $(25 \mu \mathrm{g})$, Gentamycin $(10 \mu \mathrm{g})$, Ofloxacin $(5 \mu \mathrm{g})$, Cotrimoxazole $(25 \mu \mathrm{g})$ and Nalidixic acid $(30 \mu \mathrm{g})$. It was generally observed that ofloxacin and gentamycin were more effective while cotrimoxazole and amoxycilin were the most resisted in vitro, during the study. It is therefore, important to monitor UTI, associated pathogens and their pattern of susceptibility to different antimicrobial agents for better management of cases.
\end{abstract}

Volume 6 Issue 2 - 2018

\author{
Hamza Sule,' Auwal Uba, ${ }^{2}$ Abdulhadi Sale \\ Kumurya \\ 'Department of Medical Laboratory Science, Bayero University \\ Kano, Nigeria \\ ${ }^{2}$ Biology Programme, Abubakar Tafawa Balewa University, \\ Nigeria
}

Correspondence: Hamza Sule, Department of Medica Laboratory Science, Bayero University Kano, Nigeria, Tel

+2348036062021, Email sule.hamza@yahoo.com

Received: March 01, 2018 | Published: April 17, 2018

Keywords: urinary tract, susceptibility, antimicrobial agents, uropathogens

\section{Introduction}

Urinary Tract Infection (UTI) is a general term refers to infection/ inflammation of any part of the urinary tract caused by microorganisms including bacteria. UTI is one of the most common bacterial infections encountered by clinicians worldwide. ${ }^{1}$ Moreover, since reporting of antibiotic susceptibility results in suspected cases of UTI, takes at least 48 hours following sampling, conditions are sometimes treated empirically, based on available clinical data, this sometimes leads to antibiotic resistance. ${ }^{2}$ Among the most common infectious diseases, urinary tract infections are ranked higher by clinicians in developing countries with an estimated annual global incidence of at least 250 million. ${ }^{3,4}$ Treatment of UTIs cases, is often started empirically and therapy is based on information determined from the previous antimicrobial susceptibility pattern of the urinary pathogens. ${ }^{5}$ However, a large proportion of uncontrolled antibiotic usage has contributed to the emergence of resistant bacterial infections. ${ }^{6-9}$ It is known that, the prevalence of antimicrobial resistance among urinary pathogens has been increasing worldwide. Associated resistance (the fact that a bacterium resistant to one antibiotic is often much more likely to be resistant to another) drastically decreases our chances of getting a second empirical attempt right. ${ }^{10}$

It was found that, one survey results performed in the USA estimated that a UTI episode was associated with an average of 6.1 days with symptoms, 2.4 days of reduced activity and 0.4 days of bed rest, thus generating an estimated annual cost (direct and indirect) of 1.6 billion dollars. ${ }^{11-13}$ In China, UTIs account for $9.39-50 \%$ of nosocomial infections.9 10 Most cases of UTI are caused by Gramnegative bacilli, with Escherichia coli sometimes accounting for over $90 \%$ of uncomplicated UTIs. ${ }^{14}$ Urinary Tract Infection (UTI) has become the most common hospital-acquired infection, accounting for as many as $35 \%$ of nosocomial infections, and it is the second most common cause of bacteraemia in hospitalized patients. ${ }^{15}$ It accounts for a significant proportion of the work load in clinical microbiology laboratories and enteric bacteria remained the most frequent cause, although the distribution of causative pathogens is changing by locality and by intrinsic and extrinsic factors. ${ }^{15}$

In pregnancy, cases often begins in week 6 and peaks during weeks 22 to 24 of the pregnancy, and this is due to a number of factors including urethral dilatation, increased bladder volume and decreased bladder tone, along with decreased urethral tone which contributes to increased urinary stasis and ureterovesical reflux, in which up to $70 \%$ of pregnant women develop glycosuria, and this encourages bacterial growth in the urine. ${ }^{16}$ The objective of the study was to isolate common bacteria responsible for UTI and to determine their susceptibility pattern to commonly used antimicrobial agents in Kano, Nigeria.

\section{Materials and methods}

\section{Study area and population}

Three hundred (300) patients were enrolled in the study, in the three selected hospitals, in Kano metropolis: Murtala Mohammad Specialist Hospital Kano, located at the city center along Sabon titi; Infectious Disease Hospital Kano, located along France Road leading to Katsina Road. And Sir Mohammad Sunusi Hospital Kano, located along Hadejia Road leading to Jigawa State. Most are also traders and some civil servants by occupation. Geographically, Kano metropolis is located within latitude $12^{\circ} \mathrm{N}$ and $13^{\circ} \mathrm{N}$ and longitude $8^{\circ} \mathrm{E}$ and $9^{\circ} \mathrm{E}$.

\section{Specimen collection}

Universal bottles were used for the collection of the appropriate urine samples for the study. The enrolled patients were educated on the 
type of urine they should provide (i.e. clean catch midstream urine). The first passed urine may usually contain contaminants, this signifies the reason for the use of mid stream urine. All samples collected were processed immediately. But where delay was anticipated, samples were refrigerated at $8^{\circ} \mathrm{C}$ before processing.

\section{Specimen processing}

Immediately samples were collected, macroscopic examination was carried out for observable features as, colour, turbidity and blood tinge as the case may be. The uncentrifuged urine samples were mixed by rotating the container before inoculating on to Cystein Lactose Electrolyte Deficient Agar (CLED) and Blood Agar by streak method and later incubated at $37^{\circ} \mathrm{C}$ for 24 hours. ${ }^{17}$ Media used were from Titan Biotech Ltd, Delhi, India while blood agar was made from to $25 \mathrm{ml}$ of sterile sheep blood to $500 \mathrm{ml}$ nutrient agar according to Cheesbrough. ${ }^{17}$

\section{Isolation of the uropathogens}

Positive plates after 24 hours incubation were subcultured on to MacConkey agar plate to obtain discrete colonies for further characterization and identification.

\section{Identification of the isolates}

The discrete colonies obtained on purity plates were used for the identification of the isolates using standard methods and Identification was based on colonial morphology and biochemical characteristics. Colonies produced after 24 hours (on purity plate) were examined for morphological features. ${ }^{18}$

\section{Characterization of the isolates}

In addition to morphological features of the colonies obtained on the purity plates; motility, Gram staining reaction, and biochemical tests were used in characterizing the isolates according to Cheesebrough, ${ }^{17}$ as follows.

\section{Gram's staining technique}

Discrete colonies were used for the technique as follows:

i. Smears were made on a clean grease-free slides, air dried and fixed using gentle flame.

ii. The smears were flooded with crystal violet for 30 seconds.

iii. The smears were then washed with water and flooded with Gram's iodine for 1 minute.

iv. The smears were washed with water again and decolorized briefly with acetone

v. It was washed with water and counter stained for 1 minute using neutral red

vi. The slide was washed with water, drain-dried on slide rack and examined with immersion objective.

\section{Results}

Dark Purple reaction was considered as gram positive. Pale-dark red reaction was considered as gram negative.

\section{Catalase test}

Two $\mathrm{ml}$ of $3 \%$ hydrogen peroxide solution was pipetted in to three test tubes (for test, positive and negative controls). A sterile wooden stick was used to remove several colonies of the test organisms and immersed into the test tube while a known staphylococcus and streptococcus spp. were immersed into positive and negative controls tubes respectively.

\section{Results}

Bubble production in the test and positive control indicated a positive result.

\section{Coagulase test (slide method) for bound coagulase}

i. A drop of distilled water was placed on to each side of a clean grease-free slide.

ii. A colony of the test organism was emulsified in each of the two drops and a thick suspension formed.

iii. A loopful of plasma was added and mixed gently and clumping was checked within 10 seconds as a positive result.

\section{Tube method (for free coagulase)}

i. Three test tubes were labelled test, positive and negative controls.

ii. Zero point two $(0.2) \mathrm{ml}$ of plasma was added to each tubes

iii. Zero point eight $(0.8) \mathrm{ml}$ of $24 \mathrm{hr}$ broth culture of test organism, $\mathrm{S}$. aureus and sterile nutrient broth were pipetted into the tubes as test, positive and negative controls respectively, and were mixed gently.

iv. The tubes were incubated at $37^{\circ} \mathrm{C}$ and examined at $1 \mathrm{hr}$, if no clotting, was left for $3 \mathrm{hrs}$, then overnight at room temperature.

\section{Results}

Clots or fibrin dots in test and positive control tubes and none in sterile broth tube signified positive test.

\section{Indole test}

An overnight broth culture was used for the test of indole production using kovac's reagent. A clean sterilized test tube was used to transfer the overnight broth culture and about $0.5 \mathrm{ml}$ of kovac's reagent was added and shacked gently. Results were read immediately. A known E. coli and klebsiella spp. were used as positive and negative controls.

\section{Results}

A red ring on top of the test and positive control tubes and absent in the negative control tube, indicated a positive test.

\section{Oxidase test}

A clean piece of filter paper was placed in a clean petridish. Three drops of a freshly prepared oxidase reagent was applied. Using a clean wooden stick, colonies of the test organisms were removed and smeared on to the filter paper. Results were read within 10 seconds Pseudomonas and E. coli were used as positive and negative controls. 


\section{Results}

Blue-purple colour indicated positive test while no blue-purple colour indicated negative test.

\section{Citrate utelization test}

The test organisms were streaked on to slope and stab inoculated into the simon's citrate agar and incubated at $37^{\circ} \mathrm{C}$ for 24 hours with the cap loosen. Known Klebsiella and Salmonella spp. were used as positive and negative controls respectively.

\section{Results}

Bright blue medium after incubation, positive while no change in medium colour showed a negative test.

\section{Urease test}

The test organisms were streaked on to urea slope and stab inoculated into the urea agar and incubated at $37^{\circ} \mathrm{C}$ for 24 hours with the cap loosen. Known proteus and E. coli spp. were used as positive and negative controls respectively.

\section{Results}

Change of medium colour to pink was a positive test while if no colour change was a negative test.

\section{Suger test}

Kligler iron agar (KIA) were streaked with the test organisms (on the slope) and stab inoculated into the media and incubated at $37^{\circ} \mathrm{C}$ and results read after 24 hours.

\section{Results}

A yellow butt and slope indicated fermentation of both glucose and lactose. A characteristic of both E. coli and Klebsiella pneumonia which were differentiated with motility and indole tests.

\section{Motility test (by hanging drop method)}

In each test, using paraffin wax, a ring was made at the center of cover slip. A drop of the overnight broth culture was placed at the center of the ring. A clean grease-free slide was placed on the ring and quickly but gently inverted with the cover slip upper most. The slide was examined using 10x and 40x objective. E. coli and klebsiella species were used as positive and negative controls respectively.

\section{Determination of antimicrobial susceptibility pattern of the bacterial isolates}

The susceptibility pattern of the isolates to commonly used antimicrobial agents was determined using National Committee for Clinical Laboratory Standard (NCCLS) modified Kirby-Bauer disc diffusion method, (NCCLS, 2000). Discs of known concentration of antimicrobials, gentamycin $10 \mu \mathrm{g}$, nitrofurantoin $200 \mu \mathrm{g}$, nalidixic acid $30 \mu \mathrm{g}$, tetracycline $25 \mu \mathrm{g}$, a $\mu$ gumentin $30 \mu \mathrm{g}$, cotrimoxazole $25 \mu \mathrm{g}$, amoxicillin $25 \mu \mathrm{g}$, and ofloxacin $5 \mu \mathrm{g}$ were placed on plates of Muller Hinton Agar uniformly inoculated with the test organisms. Zone of inhibition around each antibiotic disc was measured using millimeter ruler (Figure 1). ${ }^{18}$

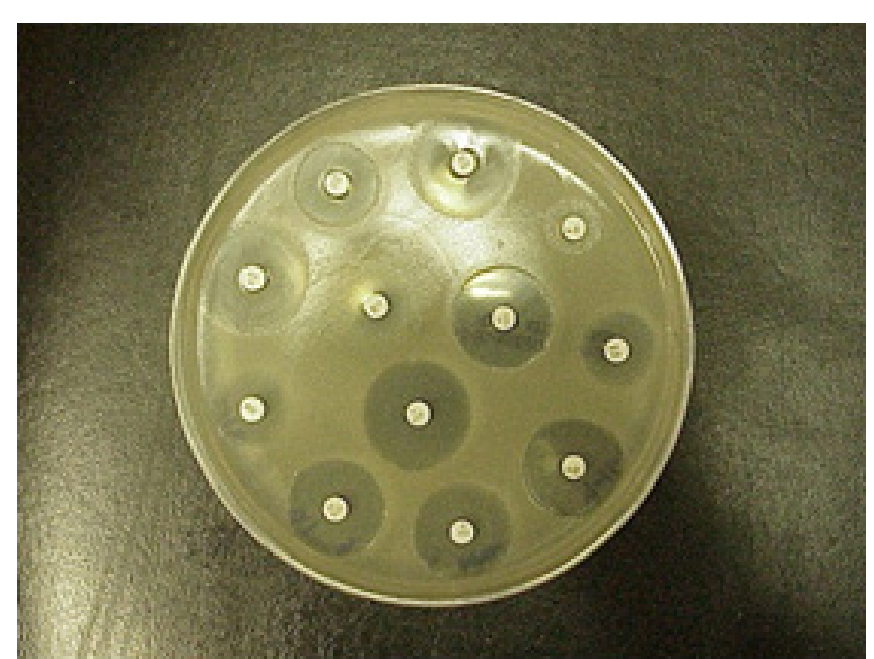

Figure I Susceptibility pattern using disc diffusion method.

\section{Data analysis}

The data obtained were analyzed using statistical package for social sciences SPSS version 20

\section{Results}

Of the total samples analyzed in the study (300), one hundred and fourteen (114), representing (38\%) were positive for culture. Different species of uropathogens were isolated with $E$. coli, as the most prevalent, 58(50.9\%), followed by Klebsiella pnuemoniae 27(23.7\%), Staphylococcus saprophyticus 11(9.6), Staphylococcus aureus 8(7.0), Proteus mirabilis $8(7.0 \%)$, and Pseudomonas aeruginosa having the least frequency of occurrence $2(1.8 \%)$ as seen Table 1.

The susceptibility pattern of the uropathogens with respect to the used antimicrobial agents has been variable (Table 2). Among the species isolated, E. coli had high sensitivity to ofloxacin $(55.1 \%)$ and higher resistance to cotrimoxazole (93.1\%), (Table 3). Klebsiella peumoniae, was found to be highly sensitive to ofloxacin (77.8\%) while mostly resisted cortrimoxazole $(81.5 \%)$ as seen in Table 4 . For Staphylococcus aureus, ofloxacin and gentamicin were found to be the most effective agents with $(12.5 \%)$ and $(25 \%)$ resisted respectively (Table 5). Ofloxacin was found sensitive, moderately sensitive and resistant, $(63.6 \%),(27.3 \%)$ and $(09.1 \%)$ to $S$. saprophyticus isolates respectively (Table 6). Proteus mirabilis on the other hand, exhibited moderate sensitivity to augumentin, amoxycillin and cotrimoxazole with 2(25.0\%) 3(37.5\%) 1(12.5\%) respectively (Table 7). The isolates of Pseudomonas aeruginosa appeared either resistant or moderately sensitive to all the antimicrobials used, (Table 8). The overall susceptibility of the isolates revealed that ofloxacin had the highest in vitro activity against most of the isolates while the second most effective under the same condition was gentamicin. Resistance was mostly observed in cotrimoxazole and amoxycillin. 
Table I Bacterial isolates obtained from the urine samples 100

\begin{tabular}{lcc}
\hline Species isolated & Frequency of occurrence & Percentage (\%) \\
\hline Escherichia coli & 58 & 50.9 \\
Klebsiella pneumoniae & 27 & 23.7 \\
Staphylococcus aureus & 8 & 7 \\
Staphylococcus saprophyticus & 11 & 9.6 \\
Proteus mirabilis & 8 & 7 \\
Pseudmonas aeruginosa & 2 & 1.8 \\
Total & 114 & 100 \\
\hline
\end{tabular}

Table 2 Antibiotic susceptibility pattern of the uropathogens in Kano, Nigeria

\begin{tabular}{lcccccccc}
\hline Aug(\%) & Nit(\%) & Amox(\%) & Tet(\%) & Gen(\%) & Oflox(\%) & Cot(\%) & Nal(\%) \\
\hline Uropth E. coli & $7(I 2.1)$ & $25(43.1)$ & $I 7(29.3)$ & $28(48.3)$ & $32(55.2)$ & $47(8 I .0)$ & $4(6.9)$ & $19(32.8)$ \\
Kleb.pneu & $9(33.3)$ & $I 2(44.4)$ & $9(33.3)$ & $I I(40.7)$ & $I 5(55.5)$ & $21(77.7)$ & $5(18.5)$ & $10(37.0)$ \\
S. aureus & $4(50.0)$ & $5(62.5)$ & $3(37.5)$ & $5(62.5)$ & $6(75.0)$ & $7(87.5)$ & $2(25.0)$ & $4(50.5)$ \\
S. saproph. & $7(63.6)$ & $8(72.7)$ & $7(63.6)$ & $7(63.6)$ & $9(81.8)$ & $10(90.9)$ & $5(45.5)$ & $5(45.5)$ \\
P. mirabilis & $2(25.0)$ & $4(50.0)$ & $3(37.5)$ & $4(50.0)$ & $5(62.5)$ & $6(75.0)$ & $I(12.5)$ & $4(50.0)$ \\
P. aeruginosa & $0(00.0)$ & $I(50.0)$ & $0(00.0)$ & $0(00.0)$ & $I(50.0)$ & $2(100.0)$ & $0(00.0)$ & $0(00.0)$
\end{tabular}

Aug, augumentin; Nit, nitrofurantoin; Amox, amoxycillin; Tet, tetracycline; Gen, gentamicin; Oflox, ofloxacin; Cot, cotrimoxazole; Nal, nalidixic acid, Kleb. Pneu, klebsiella pneumoniae; S. sapproph., Staphylococcus saprophyticus.

Table 3 Antibiotic susceptibility pattern of the Uropathogenic Eschericia coli isolated $\mathrm{N}=(58)$

\begin{tabular}{lllll}
\hline Antimicrobials & Sensitive & Intermediate & Resistance & N (\%) \\
\hline Augumentin $(30 \mu \mathrm{g})$ & - & $7(I 2.1)$ & $5 I(87.9)$ & $58(100)$ \\
Nitrofurontoin $(200 \mu \mathrm{g})$ & $8(13.8)$ & $17(29.3)$ & $33(56.9)$ & $58(100)$ \\
Amoxycillin $(25 \mu \mathrm{g})$ & $2(3.4)$ & $15(25.9)$ & $4 I(70.7)$ & $58(100)$ \\
Tetracyclin $(25 \mu \mathrm{g})$ & $7(12.1)$ & $2 I(36.2)$ & $30(51.7)$ & $58(100)$ \\
Gentamicin $(10 \mu \mathrm{g})$ & $10(17.2)$ & $22(38.0)$ & $26(44.8)$ & $58(100)$ \\
Ofloxacin $(5 \mu \mathrm{g})$ & $32(55.1)$ & $15(25.9)$ & $1 \mathrm{I}(19.0)$ & $58(100)$ \\
Cotrimoxazole $(25 \mu \mathrm{g})$ & - & $4(6.9)$ & $54(93.1)$ & $58(100)$ \\
Nalidixic acid $(30 \mu \mathrm{g})$ & $4(6.9)$ & $15(25.9)$ & $39(67.2)$ & $58(100)$ \\
\hline
\end{tabular}

Table 4 Antibiotic susceptibility pattern of the Klebsiella pneumoniae isolated $\mathrm{N}=(27)$

\begin{tabular}{lllll}
\hline Antimicrobials & Sensitive & Intermediate & Resistance & N (\%) \\
\hline Augumentin $(30 \mu \mathrm{g})$ & $3(1 \mathrm{I} .1)$ & $6(22.2)$ & $6(22.2)$ & $27(100)$ \\
Nitrofurontoin $(200 \mu \mathrm{g})$ & $3(1 \mathrm{I} .1)$ & $9(33.3)$ & $15(55.6)$ & $27(100)$ \\
Amoxycillin $(25 \mu \mathrm{g})$ & - & $9(33.3)$ & $18(66.7)$ & $27(100)$ \\
Tetracyclin $(25 \mu \mathrm{g})$ & $3(1 \mathrm{I} .1)$ & $8(29.6)$ & $16(59.3)$ & $27(100)$ \\
Gentamicin $(10 \mu \mathrm{g})$ & $7(26.0)$ & $8(29.6)$ & $12(44.4)$ & $27(100)$ \\
Ofloxacin $(5 \mu \mathrm{g})$ & $15(55.6)$ & $6(22.2)$ & $6(22.2)$ & $27(100)$ \\
Cotrimoxazole $(25 \mu \mathrm{g})$ & - & $5(18.5)$ & $22(81.5)$ & $27(100)$ \\
Nalidixic acid $(30 \mu \mathrm{g})$ & $4(14.8)$ & $6(22.2)$ & $17(63.0)$ & $27(100)$ \\
\hline
\end{tabular}


Table 5 Antibiotic susceptibility pattern of the staphylococcus aureus isolated $(\mathrm{N}=8)$

\begin{tabular}{lllll}
\hline Antimicrobials & Sensitive & intermediate & Resistance & N (\%) \\
\hline Augumentin $(30 \mu \mathrm{g})$ & $2(25.0)$ & $2(25.0)$ & $4(50.0)$ & $8(100)$ \\
Nitrofurontoin $(200 \mu \mathrm{g})$ & $\mathrm{I}(12.5)$ & $4(50.0)$ & $3(37.5)$ & $8(100)$ \\
Amoxycillin $(25 \mu \mathrm{g})$ & $\mathrm{I}(12.5)$ & $2(25.0)$ & $5(62.5)$ & $8(100)$ \\
Tetracyclin $(25 \mu \mathrm{g})$ & $2(25.0)$ & $3(37.5)$ & $3(37.5)$ & $8(100)$ \\
Gentamicin $(10 \mu \mathrm{g})$ & $4(50.0)$ & $2(25.0)$ & $2(25.0)$ & $8(100)$ \\
Ofloxacin $(5 \mu \mathrm{g})$ & $5(62.5)$ & $2(25.0)$ & $1(12.5)$ & $8(100)$ \\
Cotrimoxazole $(25 \mu \mathrm{g})$ & - & $2(25.0)$ & $6(75.0)$ & $8(100)$ \\
Nalidixic acid $(30 \mu \mathrm{g})$ & - & $4(50.0)$ & $4(50.0)$ & $8(100)$ \\
\hline
\end{tabular}

Table 6 Antibiotic susceptibility pattern of the $S$. saprophyticus isolaled $(\mathrm{N}=\mathrm{II})$

\begin{tabular}{lllll}
\hline Antimicrobials & Sensitive & intermediate & Resistance & N (\%) \\
\hline Augumentin $(30 \mu \mathrm{g})$ & $2(18.2)$ & $5(45.4)$ & $5(45.4)$ & $\mathrm{II}(\mathrm{I00})$ \\
Nitrofurontoin $(200 \mu \mathrm{g})$ & $2(18.5)$ & $6(54.5)$ & $3(27.3)$ & $\mathrm{II}(\mathrm{I00})$ \\
Amoxycillin $(25 \mu \mathrm{g})$ & $3(27.2)$ & $4(36.4)$ & $4(36.4)$ & $\mathrm{II}(100)$ \\
Tetracyclin $(25 \mu \mathrm{g})$ & $4(36.4)$ & $3(27.2)$ & $4(36.4)$ & $\mathrm{II}(\mathrm{I00})$ \\
Gentamicin $(10 \mu \mathrm{g})$ & $5(45.4)$ & $4(36.4)$ & $2(18.0)$ & $\mathrm{II}(100)$ \\
Ofloxacin $(5 \mu \mathrm{g})$ & $7(63.6)$ & $3(27.3)$ & $\mathrm{I}(09 . \mathrm{I})$ & $\mathrm{II}(100)$ \\
Cotrimoxazole $(25 \mu \mathrm{g})$ & - & $5(45.5)$ & $6(54.5)$ & $\mathrm{II}(100)$ \\
Nalidixic acid $(30 \mu \mathrm{g})$ & $\mathrm{I}(9.1)$ & $4(36.4)$ & $6(54.5)$ & $\mathrm{II}(100)$ \\
\hline
\end{tabular}

Table 7 Antibiotic susceptibility pattern of the Proteus mirabilis isolated N= (8)

\begin{tabular}{lllll}
\hline Antimicrobials & Sensitive & intermediate & Resistance & N (\%) \\
\hline Augumentin $(30 \mu \mathrm{g})$ & - & $2(25.0)$ & $6(75.0)$ & $8(100)$ \\
Nitrofurontoin $(200 \mu \mathrm{g})$ & $\mathrm{I}(\mathrm{I} 2.5)$ & $3(37.5)$ & $4(50.0)$ & $8(100)$ \\
Amoxycillin $(25 \mu \mathrm{g})$ & - & $3(37.5)$ & $5(62.5)$ & $8(100)$ \\
Tetracyclin $(25 \mu \mathrm{g})$ & $2(25.0)$ & $2(25.0)$ & $4(50.0)$ & $8(100)$ \\
Gentamicin $(10 \mu \mathrm{g})$ & $3(37.5)$ & $2(25.0)$ & $3(37.5)$ & $8(100)$ \\
Ofloxacin $(5 \mu \mathrm{g})$ & $4(50.0)$ & $2(25.0)$ & $2(25.0)$ & $8(100)$ \\
Cotrimoxazole $(25 \mu \mathrm{g})$ & - & $\mathrm{I}(12.5)$ & $7(87.5)$ & $8(100)$ \\
Nalidixic acid $(30 \mu \mathrm{g})$ & $\mathrm{I}(\mathrm{I} 2.5)$ & $3(37.5)$ & $4(50.0)$ & $8(100)$
\end{tabular}

Table 8 Antibiotic susceptibility pattern of the Pseudomonas aeruginosa Isolated $\mathrm{N}=(2)$

\begin{tabular}{lllll}
\hline Antimicrobials & Sensitive & Intermediate & Resistance & N (\%) \\
\hline Augumentin $(30 \mu \mathrm{g})$ & - & $2(25.0)$ & $2(100)$ & $2(100)$ \\
Nitrofurontoin $(200 \mu \mathrm{g})$ & - & $\mathrm{I}(50.0)$ & $\mathrm{I}(50.0)$ & $2(100)$ \\
Amoxycillin $(25 \mu \mathrm{g})$ & - & - & $2(100)$ & $2(100)$ \\
Tetracyclin $(25 \mu \mathrm{g})$ & - & - & $2(100)$ & $2(100)$ \\
Gentamicin $(10 \mu \mathrm{g})$ & - & $\mathrm{I}(50.0)$ & $\mathrm{I}(50.0)$ & $2(100)$ \\
Ofloxacin $(5 \mu \mathrm{g})$ & - & $2(100)$ & - & $2(100)$ \\
Cotrimoxazole $(25 \mu \mathrm{g})$ & - & - & $2(100)$ & $2(100)$ \\
Nalidixic acid $(30 \mu \mathrm{g})$ & - & - & $2(100)$ & $2(100)$ \\
\hline
\end{tabular}




\section{Discussion}

The result of the study indicated that, E. coli and Klebsiella pneumonia had the highest prevalence rate (50.9\%) and (23.7\%) while the only Gram positive bacteria isolated were Staphylococcus aureus and Staphylococcus saphrophyticus $(7.0 \%)$ and $(9.6 \%)$ respectively. Similar pattern of uropathogens representation was found Getenet et al., ${ }^{19}$ in a study titled: Bacterial Uropathogens in Urinary Tract Infection and Antibiotic Susceptibility Pattern in Jimma University Specialized Hospital, Southwest Ethiopia with (33.3\%) and (19.0\%) for the highest isolates (E. coli and Klebsiella pneumonia) while Staphylococcus aureus and Staphylococcus saprophyticus were $(4.8 \%)$ and $(14.3 \%)$ respectively. While the two studies exhibits similar isolation pattern, the percentage occurrence of the pathogen varied. These isolates percentages variations could be attributable to geographical differences between the two study areas. However a similar study in China, ${ }^{20}$ revealed a different isolation pattern and species and strains diversity, because, while we had E. coli and Klebsiella pneumoniae as the predominant uropathogens in this study $(50.9 \%)$ and $(23.7 \%)$ respectively their study recorded E. coli and Staphylococcus epidemidis as organisms with highest frequency $(50 \%)$ and (9\%) respectively while Klebsiella pneumonae represented only $(5 \%)$ of the isolates. They also had more strains of the respective species isolated than found in this study, example, Staphylococcus hominis, Staphylococcus haemolyticus, Staphylococcus cleife and Staphylococcus wameri; Proteus vulgaris; Psedomonas putida; were additional strains isolated among others in their study. According to the results obtained ofloxacin and gentamicin, were the most effective agent against $E$. coli, with $81 \%$ and $55.2 \%$ of their isolates, susceptible to the agents respectively. A study in Kenyan, with title, Isolation and antimicrobial susceptibility testing of Escherichia coli causing urinary tract infections by Kebira et al. ${ }^{21}$ also had the same antimicrobial agents among the most effective against $E$. coli in vitro. The study also revealed ofloxacin as the most effective agent in vitro with $81.0 \%, 77.7 \%, 87.5 \%, 90.0 \%, 75.0 \%$ and $100 \%$ efficacy rates on, E. coli, Klebsiella pneumonia, Staphylococcus aureus, Staphylococcus saprophyticus, Proteus mirabilis, and Pseudomanas aeruginosa respectively. Cotrimoxazole was found the most resisted with only $(0.00 \%)$ and $(6.9 \%)$ efficacy rates on Pseudomonas and E. coli, but it was mildly effective against, Proteus mirabilis (12.5\%), Klebsiella pneumonia 18.1\%, Staphylococcus aureus and Staphylococcus saphyropiticus $25 \%$ and $45 \%$ respectively. A similar result was obtained in the same study area Aminu Kano Teaching Hospital, Kano, Nigeria, Chedi et al. ${ }^{22}$ in a seven months retrospective study on urinary tract infection among patients attending the hospital. In their study, fluoroquinolones to which ofloxacin (the most effective agent in our study) belongs proved the most effective class of antimicrobial agents, according to their observation, with $(73.5 \%)$, (76.9\%), (90.9\%), 78.9\% as percentage efficacy on E. coli, Proteus $s p$, Klebsiella $s p$ and Pseudomanas $s p$ respectively while almost all the isolates resisted cotrimoxazole with only $(4.8 \%)$ recorded efficacy against E. coli.

On the species individual bases, eighty one per cent (81\%) of $E$. coli isolates were sensitive to ofloxacin, $(55.2 \%)$ to gentamicin as the highest efficacious agent while amoxacillin and cotrimoxazole was effective to only $(29.3 \%)$ and $(6.9 \%)$ respectively. A North-eastern Nigerian study (Yola, Adamawa state, Nigeria) entitled, Etiology and Antimicrobial Resistance Pattern of Bacterial Agents of Urinary Tract
Infections in Students of Tertiary Institutions in Yola Metropolis, ${ }^{23}$ exhibited concurrent report on sensitivity to these agents, by the uropathogenic E. coli, with, ofloxacin (100\%), gentimicin, (94\%), amoxicillin (11\%), cotrimoxazole, (17\%). Klebsiella pneumoniae was found to be sensitive with the following isolates percentages to different antimicrobial agents (33.3\%) amoxicillin, (40.7\%) tetracyclin, (55.6\%) gentamicin, (44.4\%) nitrofurantoin, and 77.8\% ofloxacin. In the same vein, Samiah HS Al-Mijal ${ }^{24}$ in Riyadh, Saudi Arabia, had amoxcilin 84\% resisted (impying only 16\% sensitivity) by the Klebsiella pneumonia isolates, which is even lower than found in this study, nitrofurantoin which was $44.4 \%$ in this study also ended up $16 \%$ while gentamicin $55.6 \%$ in our work had $28 \%$, ofloxacin was substituted by another floroquinolone, levofloxacin and both proved to be effective against the intended uropathogens, $77.8 \%$ and $80.0 \%$ respectively. These relative differences in some parts of the two studies may be associated with local peculiarities in the respective locations which could also lead to stains variations.

Staphylococcus aureus also showed variable resistant pattern with respect to the used antimicrobials. Resistant rate were found to be, $(25 \%),(37.5 \%),(75 \%)$, and $(62.5 \%)$ for gentamicin, Tetracyclin, cotrimoxazole, and amoxicillin respectively. This implies highest

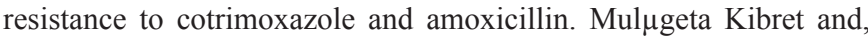
Bayeh Abera, (2014) reported a concurrent findings in Ethopia, which showed genamicin (16.7\%) resisted tetracyclin, (66.7\%) resisted, cotrimoxazole (77.8\%) resisted while amoxicillin was even $(100 \%)$ accordingly, the result therefore implies cotrimoxazole and amoxicillin as the most resisted, while gentamicin as one of the most effective as found in this study.

In particular, the resistance to cotrimoxazole are somewhat, much similar, in both cases $(75 \%)$ and $(77.8 \%)$ as indicated respectively, meaning that the agent is weakly effective against the intended organisms, but gentamicin which was the second most efficacious in vitro in this study, was also found effective based on their work, with resistance rate of $25 \%$ and below, which corresponds to $75 \%$ and above sensitivity to the agents. Getenet Beyene et al. ${ }^{19}$ reported from University Specialized Hospital, Southwest Ethiopia, a higher sensitivity rate than found in this study, by Staphylococcus sapphyropiticus isolates, in which genytamicin, tetracyclin, and nitrofurantoin were $0 \%$ resisted (meaning $100 \%$ sensitivity) as against $18 \%, 36.4 \%$ and $(27.3 \%)$ respectively, nalidixic acid had $(2 \%)$ resisted as against $(54.5 \%)$ while amoxicillin was $(66.7 \%)$ as against (36.4\%) for them and our study respectively.

The $100 \%$ sensitivity to many of the tested agents may suggest a less degree of misuse and abuse of drugs while higher resistance indicates the contrary in the respective study areas. Acording to the study, Proteus mirabillis exhibited the following susceptibility pattern, amoxicilin $(37.5 \%)$, tetracyclin $(50 \%)$, cortrimoxazole $(12.5 \%)$, oflooxacin $(75 \%)$ and nitrofurantoin $(50 \%)$. These indicated highest percentage effectivity by ofloxacin followed by nitrofurantoin and respectively, the same agents were also found as most effective in a study conducted in university of Benin, Orhue, ${ }^{25}$ ofloxaxacin $(64.8 \%)$ and nitrofurantoin ( $58.6 \%$ ), however, there were some variations in terms of effectiveness of some of the agents used, example, while tetracyclin was active against $(50 \%)$ of the isolates in our study, it proved active on only (17.9\%) of isolates in the Benin study. Similarly, cotrmoxazole was less active in vitro according to our study while it was discovered moderately promising in their work 
$(12.5 \%)$ and $(42.9 \%)$ respectively. In vitro activity of amoxicillin was not encouraging in both studies, because, $(37.5 \%)$ and $(21.4 \%)$ of isolates were respectively resisted.

In this study, Pseudomonas was found to be (100\%) resistant to nalidixic acid, augumentin and cotrimoxazole while (50\%) resistant to gentamicin, on the other hand found (100\%) sensitive to ofloxicin, Shah et al., ${ }^{26}$ also found (99.2\%), (98.8\%) and (97.6\%) for cortrimoxazole, nalidixic acid and augumentin while gentamicin and ofloxacin were found only (35.3\%) and (49\%) resisted respectively. The (49\%) resistant to ofloxacin could be, because the drug use to be effective, it were over prescribed in the area, which led to the development of resistant strains. ${ }^{27}$

\section{Conclusion}

This study also showed that ofloxacin and gentamicin were the most active antibiotics against uropathogens. Thus it is believed that these antibiotics should be used in the treatment of Urinary tract infection in this region. Moreover, this study has provided epidemiologic data and there is the need for consistent on-going antimicrobial resistance surveillance for important and commonly isolated clinically significant uropathogens to form the basis for developing and implementing measures that can reduce the burden of antimicrobial resistance and prevent a probable impending public health problem.

\section{Recommendation}

Government should put more strict rules on the sale of antibiotics and awareness campaign on the significance of consulting doctor before taking medication and completing regimens when prescribed should be made. Ofloxacin and gentamicin should be used to manage Urinary tract infection in Kano, Nigeria.

\section{Acknowledgements}

We acknowledged the Management of the hospitals used in study for the given and for providing enabling environment to carry out the study.

\section{Conflict of interest}

The author declares no conflict of interest.

\section{References}

1. Biadglegn F, Abera B. Antimicrobial resistance patterns of bacterial isolates from urinary tract infections at Felege Hiwot Referral Hospital, Ethiopia. Ethiop J Health Dev. 2009;23:236-238.

2. Akoachere JF, Yvonne S, Akum NH, et al. Etiologic profile and antimicrobial susceptibility of community-acquired urinary tract infection in two Cameroonian towns. BMC Res Notes. 2012;5:219.

3. Ronald AR, Nicolle LE, Stamm E. Urinary tract infection in adults: research priorities and strategies. Int $J$ Antimicrob Agents. 2001;17(4):343-348.

4. Barisic Z, Babic-Erceg A, Borzic E, et al. Urinary tract infections in South Croatia: aetiology and antimicrobial. Intl $J$ Antimicrob Agents. 2003;22(Suppl 2):61-64

5. Wilson ML, Gaido L. Laboratory Diagnosis of Urinary Tract Infections in Adult Patients. Clin Infect Dis. 2004;38(8):1150-1158.
6. Bonadio M, Meini M, Spetaleri P, et al. Current microbiological and clinical aspects of urinary tract infections. Eur J Urol. 2001;40(4):439445 .

7. National Committee for Clinical Laboratory Standards. Performance standards for antimicrobial disc susceptibility tests. 7th ed. Wayne, Pennsylvania, USA: NCCLS; 2000.

8. Grude N, Tveten Y, Kristiansen BE. Urinary tract infections in Norway: bacterial etiology and susceptibility, a retrospective study of clinical isolates. Clin Microbiol Infect. 2001;7(10):543-547.

9. Kripke C. Duration of therapy for women with uncomplicated UTI. Am Fam Physician. 2005;72(11):2219.

10. Sundqvist M, Kahlmeter G. Pre-emptive culturing' will improve the chance of getting it right' when empirical th erapy of urinary tract infections fails. J Antimicrob Chemother. 2009;64(2):227-228.

11. Foxman B, Gillespiem B, Koopman J. Risk factors for second urinary tract infection among college women. Am J Epidemiol. 2000;151:1194205.

12. Foxman B, Frerichs RR. Epidemiology of urinary tract infection. II. Diet, clothing, and urination habits. Am J Public Health. 1985;75:1314-1317.

13. Foxman B. Epidemiology of urinary tract infections: incidence, morbidity and economic costs. Am J Med. 2000;113(Suppl 1A):S5-13.

14. Talan D, Stamm W, Hooton T. Comparison of ciprofloxacin (7days) and trimethoprim-sulfamethoxazole (14days) for acute uncomplicated pyelonephritis in women: a randomized trial. JAMA. 200;283(12):15831590.

15. Epoke CO, Anyanwu GO, Opara AA. The Prevalence of significant bacteriuria in diabetic patients. Diabetic International.2000;10:16-17.

16. Van Brummen HJ, Bruinse HW, van der Bom JG, et al. How do the prevalences of urogenital symptoms change during pregnancy? Neurourol Urodyn. 2006;25(2):135-139.

17. Cheesebrough M. Distric Laboratory Practice in Tropical Countries. 2nd ed. Chambridge University Press; 2000:434.

18. Mackey, McCartney. Tropical Medical Micrbiology. 14th ed. London, UK: Churchill Livingstone; 1996:978.

19. Getenet B, Wondewosen T. Bacterial Uropathogens in Urinary Tract Infection and Antibiotic Susceptibility Pattern in Jimma University Specialized Hospital, Southwest Ethiopia. Ethiop J Health Sci. 2011;21(2):141-146.

20. Lu-Dong Qiao, Shan Chen, Yong Yang, et al. Characteristics of urinary tract infection pathogens and their in vitro susceptibility to antimicrobial agents in China: data from a multicenter study. BMJ Open. 2013;3(12):e004152.

21. Kebira AN, Ochola P, Khamadi SA. Isolation and antimicrobial susceptibility testing of Escherichia coli causing urinary tract infections. Journal of Applied Biosciences. 2009;22:1320-1325.

22. Chedi BAZ, Wannang NN, Halliru MA, et al. A seven months retrospective study on urinary tract infection among patients at Aminu Kano Teaching Hospital, Kano - Nigeria. Bajopas. 2009;2(2):95-98.

23. Adedeji BAM, Abdulkadir OA. Etiology and antimicrobial resistance pattern of bacterial agents of urinary tract infections in students of tertiary institutions in yola metropolis. Academic J Plant Sci. 2009;2(1):25-28.

24. Samiah HS Al-Mijalli. Bacterial Uropathogens in Urinary Tract Infection and Antibiotic Susceptibility Pattern in Riyadh Hospital, Saudi Arabia. Cell Mol Med. 2017;3:1. 
25. Orhue OP. Antibiogram Study of Proteus spp. Bacterial Isolates from Uropathogenic Infections in University of Benin Teaching Hospital, Nigeria. Current Research in Bacteriology. 2014;7(1):12-21.

26. Shah DA, Wasim S, Abdullah FE. Antibiotic resistance pattern of Pseudomonas aeruginosa isolated from urine samples of Urinary Tract Infections patients in Karachi, Pakistan. PakJ Med Sci. 2015;31(2):341345 .
27. Mulugeta Kibret, Bayeh Abera. Prevalence and antibiogram of bacterial isolates from urinary tract infections at Dessie Health Research Laboratory, Ethiopia. Asian Pac J Trop Biomed. 2014;4(2):164-168. 